\title{
FETAL CEREBRAL HEMORRHAGE DIAGNOSED ANTEPARTUM
}

\author{
Claudiu Marginean ${ }^{1}$, Lucian Puscasiu ${ }^{1}$, Molnar V. Claudiu ${ }^{1}$, Florin Rozsnyai ${ }^{1}$, \\ Bela Szabo', Luminita Zahiu², Marta Simon², Manuela Cucerea², \\ Cristina Oana Marginean ${ }^{3}$, Nicoleta Suciu ${ }^{4}$, Cosmin Rugina ${ }^{5}$ \\ ${ }^{1}$ Obstetrics and Gynecology Clinic 1, Clinical Emergency Hospital, Targu-Mures \\ ${ }^{2}$ Neonatology Clinic 1, Clinical Emergency Hospital, Targu-Mures \\ ${ }^{3}$ Pediatrics Clinic 1, Clinical Emergency Hospital, Targu-Mures \\ ${ }^{4}$ University of Medicine and Pharmacy, Targu-Mures \\ ${ }^{5}$ Department of Obstetrics and Gynecology, Clinical Country Hospital, Piatra Neamt
}

\begin{abstract}
The diagnosis of fetal cerebral hemorrhage (HCF) is less reported $(0,9 / 1000$ births) in comparison to the postpartum one. We presented 2 cases of fetal intraventricular hemorrhages diagnosed.

Case 1: HCF diagnosed by ultrasound at the gestational age of 28 weeks and confirmed after birth by the presence of erythrocytes (significant number, pathological aspect) in the spinal fluid and by the modifications signaled by the transfontanellar ultrasound (severe internal hydrocephaly, minimum cerebral tissue). The death occurred after 24 hours postpartum (pathological exam: intraventricular cerebral hemorrhage). We mention the apparent lack of maternal risk factors, negative TORCH tests.

Case 2: internal hydrocephaly at the age of 37 gestational weeks and confirmed in newborn (after cesarean section) by the clinical characteristic modifications, severe neurological impairment with modification at the transfontanellar ultrasound and the hemorrhagic and hypertensive aspect of the spinal fluid. We did not encounter maternal risk factors. Also, apparently, there were not any fetal risks: weight 2,820 grams at 37 gestational weeks, negative TORCH tests, negative cultures and CRP. The hydrocephaly had a progressive evolution imposing ventriculotomies and cysternotomies; the placement of a ventriculoperitoneal shunt during the third neurosurgical intervention improved the vital prognosis.

Conclusions. The presence of hydrocephaly was a faithful ultrasound sign for the diagnosis of HCF. The evolution and the prognosis are influenced by the severity of hemorrhage and the gestational age. The survivors benefit from neurosurgical treatment. The multifactorial etiology, of the HCF incompletely elucidated leads to difficulties in establishing a target group of screening, in order to identify this lesion.
\end{abstract}

Keywords: cerebral hemorrhage, antepartum diagnosis

\section{INTRODUCTION}

Neonatal intracranial hemorrhage is a wellknown phenomenon, especially in preterm babies, when the most common is the ventricular or subependymal hemorrhage (1). According to Vergani, the maximal incidence is of $0.9 / 1,000$ cases among the monitored pregnancies (2). The classification of severity of fetal intraventricular hemorrhages, as well as the neonatal ones, includes: first degree - defined as limited to the subependymal matrix; second degree defined as image of ventricular hemorrhage, with less than $50 \%$ refilling of the lateral ventricle and not bigger than $15 \mathrm{~mm}$ ventriculo- megaly measured in the atrium; third degree $-50 \%$ filling or more in one or both lateral ventricles, with ventriculomegaly, but without obvious parenchymal impairment, ventriculomegaly over $15 \mathrm{~mm}$ being also included in this category; and fourth degree - manifestations included in the 1-3 degrees associated with image of hemorrhage in the periventricular parenchyma (3). The ultrasound is the most important tool in establishing the diagnosis of fetal intracranial hemorrhage. The suggestive ultrasound signs are: asymmetrical ventriculomegaly, ipsilateral thalamus atrophy, signs of restate blood in the spinal fluid, unregulated echoic areas around the ventricles or intraventricular "echoic foci", uni- 
lateral hemorrhage in the germinal matrix with cystic resorption (4). These unregulated echoic areas can be intraventricular, periventricular, subependymal, in the parenchyma, subdural, intracerebellar. Magnetic resonance is another important diagnostic method of intracranial hemorrhage which provides increased sensibility and specificity in comparison to ultrasound. The treatment of severe cases involves usually neurosurgical interventions with ventriculoperitoneal shunt placement. Regarding the mild forms, they usually require only monitoring. The evolution and prognosis of the fetal cerebral hemorrhage depends on multiple factors, especially on hemorrhage severity.

\section{CASE PRESENTATION}

We present two cases of fetal cerebral intraventricular hemorrhage diagnosed by antepartum ultrasound in the Obstetrics and Gynecology Clinic 1 from Targu-Mures.

Case 1-a 26-year-old pregnant woman, secundigesta and secundipara, without relevant pathological history before and during the pregnancy, monitored by ultrasound, with the last evaluation at 22 gestational weeks, presented for the 28 weeks check-up. At the beginning of the $3^{\text {rd }}$ trimester of pregnancy, the ultrasound pointed out unilateral ventriculomegaly, with the image of the fetal lateral cerebral ventricle with medium echogenicity, unregulated aspect, suggesting an intraventricular clot and minimum remaining cerebral tissue. After the steroid administration, we performed a cesarian section and the newborn weighted 1,200 grams, Apgar 4/1 minute. Lumbar puncture performed at 6 hours postpartum, revealed over 1,000 abnormal, deformed erythrocytes/microscopic field in the spinal fluid and confirmed the old intraventricular hemorrhage from the intrauterine life. The transfontanellar ultrasound confirmed the severe internal hydrocephaly $4^{\text {th }}$ [according to Lustig Gillman classification (3)], with minimum remaining cerebral tissue. The evolution of the intubated newborn in neonatal intensive care unit was unfavorable, with death after 24 hours postpartum, associating a severe hyaline membranes disease. Tests for identification of a TORCH syndrome were negative.

Case 2 - a 26-year-old female, first pregnancy, without pathological history, with a monitored pregnancy, ultrasound without modification at 33 gestational weeks presented for the regular checkup at 37 gestational weeks. The fetal ultrasound revealed an internal hydrocephaly, with moderate echogenicity image, unregulated in the lateral ven-
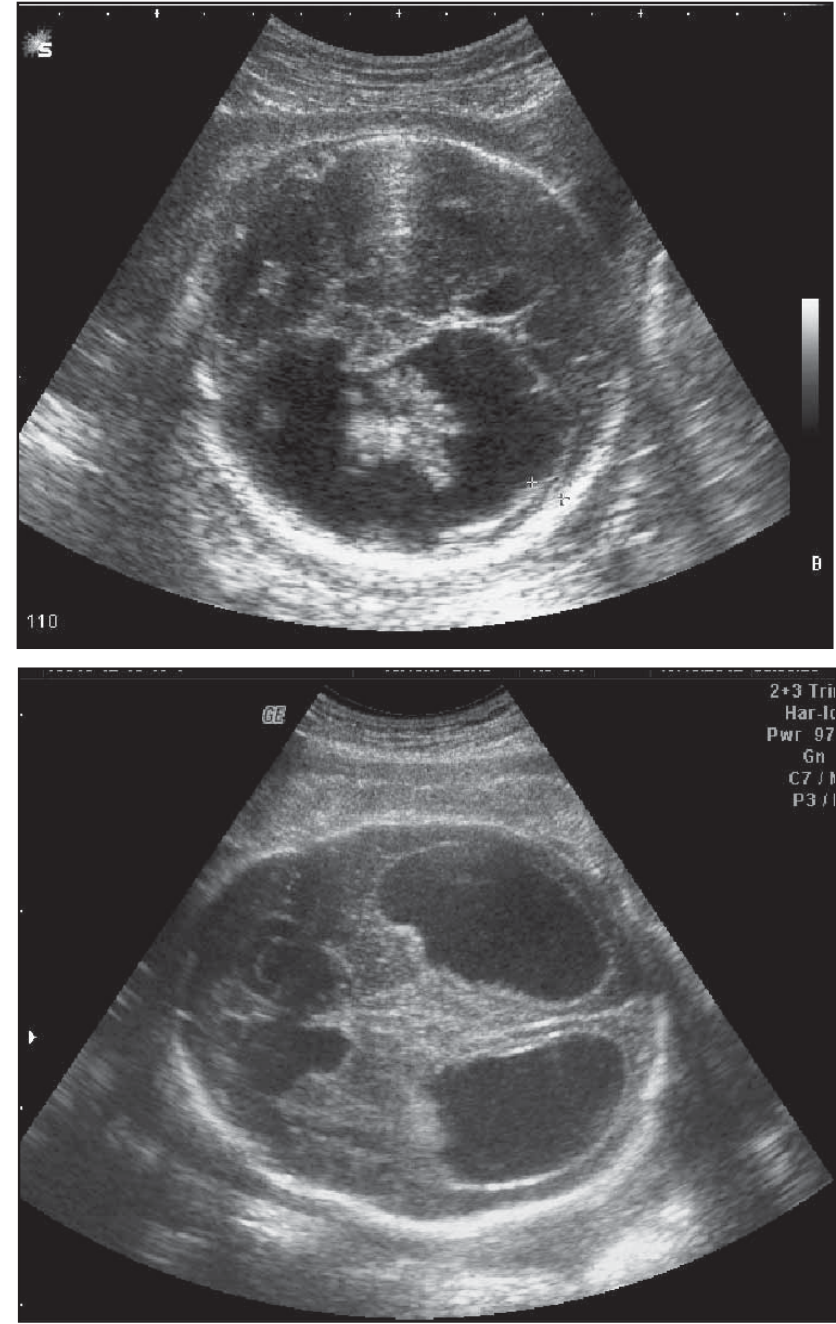

FIGURE 1. Case 1 (left image) and case 2 (right image) fetal ultrasound axial cranial section - dilated lateral ventricles and unregulated image with medium echogenicity - intraventricular hemorrhage

tricle, and breech presentation of the fetus. After 24 hours, we delivered the fetus by cesarean section. The fetal Apgar score was 9/1 minute with macrocephaly, cranial perimeter $38.5 \mathrm{~cm}$, a birth weight of 2,820 grams, anterior fontanella $5 \times 6 \mathrm{~cm}$, posterior fontanella $1 \times 5 \mathrm{~cm}$, both in tension with dehiscent sutures, newborn with tonus and reflexes impairment, diminished reactivity. Transfontanellar ultrasound $-3^{\text {rd }}$ degree right intraventricular hemorrhage [according to Lustig Gillman classification (3)], secondary hydrocephaly (diameter of the right lateral ventricle $2.6 \mathrm{~cm}$, the left one $2.0 \mathrm{~cm}$, the diameter of the $3^{\text {rd }}$ ventricle $0.7 \mathrm{~cm}$, resistivity index of the anterior cerebral artery above 1. Evacuator and diagnostic lumbar puncture, performed immediately postpartum, revealed a hypertensive hemorrhagic spinal fluid, with a number of $117,600 /$ field erythrocytes, leukocytes 514/field, bacteriological exam of the spinal fluid without bacterial growth, negative TORCH test, blood culture and CRP. The 
evolution in the following 3 months was with progressive hydrocephaly and intracranial hypertension, and he underwent 2 ventriculotomies and cysternotomies; during the $3^{\text {rd }}$ neurosurgical intervention performed abroad, a ventriculoperitoneal shunt was placed, with favorable evolution until the present moment (the last check-up at the age of 3 years).

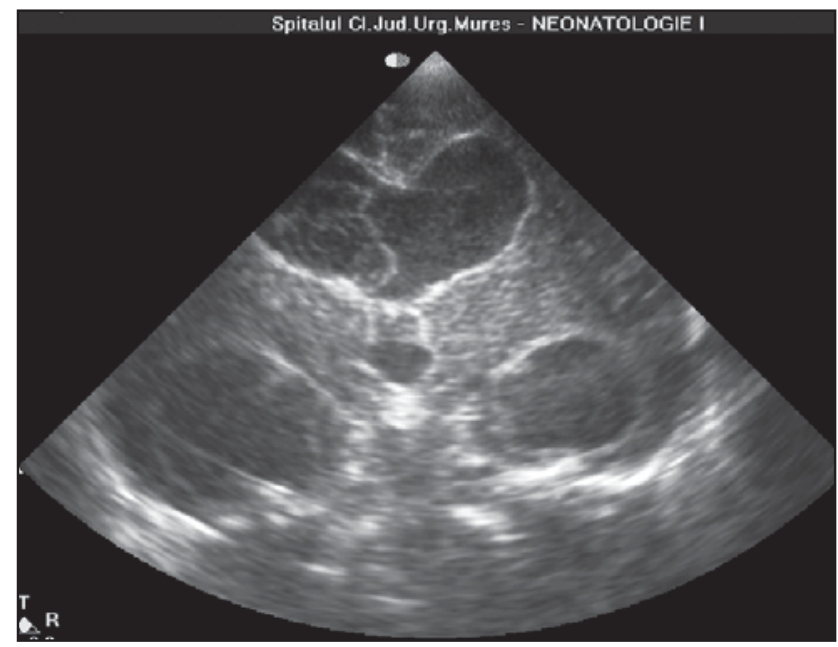

FIGURE 2. Case 2 - Postpartum transfontanellar ultrasound coronal section with obvious ventriculomegaly of the occipital horn of the lateral and $3^{\text {rd }}$ ventricle

\section{DISCUSSIONS}

The etiology of the neonatal intraventricular hemorrhage is related to: intravascular factors control of pressure inside the microcirculation from the germinal matrix, fluctuations in the cerebral flow that lead to the impairment of the placental function and coagulation; vascular factors - related to matrix microcirculation, the site of the initial hemorrhage, impairment of the matrix vessels maturation with ischemia; extravascular factors - mesenchymal and support glial factors for the matrix vessels involved in the fibrinolytic and proteolytic activity of the germinal matrix (5). The incriminated predisposing factors of the fetal intracranial hemorrhage are: maternal - idiopathic or autoimmune thrombocytopenia, von Willebrand disease, the use of warfarine or cocaine, seizures, severe abdominal trauma that involves the fetus, amniocentesis, pregnancy cholestasis, febrile diseases; or fetal ones - deficiencies of the coagulation factors $\mathrm{V}$ and $\mathrm{X}$, tumor hemorrhages, twin-to-twin transfusion syndrome, multiple pregnancy with dead fetus, feto-maternal hemorrhage $(6,7)$. In a review of the literature on 47 cases, Özduman considers that half of these fetal intracerebral hemorrhages are due to autoimmune thrombocytopenia or trauma (8). The two cases presented above did not present either autoimmune thrombocytopenia or trauma. A study performed on a group of 113 fetuses found an association between fetal intracranial hemorrhage and intrauterine growth retardation in approximately $13 \%$ of the cases (9), but in our cases we did not find this relationship. Gould et al (10), in 2005, described for the first time, in mice, the genetic mutation that can lead to cerebral "in utero" hemorrhage, namely the mutation of COL4A1 gene which is known to codify collagen 4A1 (10). COL4A1 is a gene that codifies the alfa- 1 chain of the collagen type IV, a membrane protein that is found widely in all tissues including the vessels. It seems that the impairment of this protein predisposes to hemorrhage (11-13). However, the certain involvement of this mutation in the etiology of fetal intracranial hemorrhage remains unclear according to the 2 cases presented by Garel: first fetus with intraventricular hemorrhage and the termination of the pregnancy at 30 gestational weeks, and the second one with amniocentesis and fetus with the same mutation, but without any disorder until the age of 9 months postpartum (14). Hydrocephaly is considered the most frequent ultrasound sign associated with intraventricular or subependymal signs of hemorrhage (15). The cases presented above were diagnosed by ultrasound during intrauterine life, and both presented hydrocephaly. Vergani and colab. (2) analyzing the clinical signification of the fetal intracranial hemorrhage (41 cases) correlated unfavorable evolution with the parenchymal hemorrhages (12/13), subdural and subarachnoid ones (7/8), while intraventricular hemorrhages has a mild better prognosis (11/20). On a series of 4 cases of intraventricular hemorrhage diagnosed "in utero" of $3^{\text {rd }}$ and $4^{\text {th }}$ degree, the perinatal death produced in 3 of 4 cases, the $4^{\text {th }}$ one had a normal evolution after vetriculoperitoneal shunt placement (4). From the two cases presented above, the first died shortly after birth, while the second one presented a favorable evolution after ventriculoperitoneal shunt placement.

\section{CONCLUSIONS}

The presence of hydrocephaly was a faithful ultrasound sign for the diagnosis of fetal cerebral hemorrhage. The evolution and prognosis of these lesions depend on hemorrhage severity and gestational age. The survivors benefit from the neurosurgical treatment. The multifactorial and unclear etiology of this fetal disorder leads to the difficulty in establishing a "target" screening group in order to identify these lesions. 


\section{REFERENCES}

1. Reiss I., Gortner L., Moller J., Gehl H.B., Baschat A.A., Gembruch $U$. Fetal intracerebral hemorrhage in the second trimester: diagnosis by sonography and magnetic resonance imaging. Ultrasound Obstet Gynecol Off J Int Soc Ultrasound Obstet Gynecol, 1996 Jan, 7(1):49-51.

2. Vergani P., Strobelt N., Locatelli A., Paterlini G., Tagliabue P., Parravicini E., et al. Clinical significance of fetal intracranial hemorrhage. Am J Obstet Gynecol, 1996 Sep, 175(3 Pt 1):536-43.

3. Lustig-Gillman I., Young B.K., Silverman F., Raghavendra B.N., Wan L., Reitz M.E., et al. Fetal intraventricular hemorrhage: Sonographic diagnosis and clinical implications. J Clin Ultrasound, 1983 Jun 1, 11(5):277-80.

4. Huang Y.F., Chen W.C., Tseng J.J., Ho E.S.C., Chou M.M. Fetal intracranial hemorrhage (fetal stroke): report of four antenatally diagnosed cases and review of the literature. Taiwan J Obstet Gynecol, 2006 Jun, 45(2):135-41.

5. Volpe J.J. Intraventricular hemorrhage in the premature infant-current concepts. Part I. Ann Neurol, 1989 Jan, 25(1):3-11.

6. Ramenghi L.A., Fumagalli M., Righini A., Triulzi F., Kustermann A., Mosca F. Thrombophilia and fetal germinal matrix-intraventricular hemorrhage: does it matter? Ultrasound Obstet Gynecol Off J Int Soc Ultrasound Obstet Gynecol, 2005 Oct, 26(5):574-6.

7. Lionnet C., Body G., Gold F., Paillet C., Vaillant M.C., Alle C., et al. Accident cérébral fœtal pat hémorragie fœtomaternelle massive: un cas. J Gynécologie Obstétrique Biol Reprod, 1995, 24(5):553-6.

8. Ozduman K., Pober B.R., Barnes P., Copel J.A., Ogle E.A., Duncan C.C., et al. Fetal stroke. Pediatr Neurol, 2004 Mar, 30(3):151-62.

9. Baschat A.A., Gembruch U., Viscardi R.M., Gortner L., Harman C.R. Antenatal prediction of intraventricular hemorrhage in fetal growth restriction: what is the role of Doppler? Ultrasound Obstet Gynecol Off J Int Soc Ultrasound Obstet Gynecol, 2002 Apr, 19(4):334-9.

10. Gould D.B., Phalan F.C., Breedveld G.J., van Mil S.E., Smith R.S., Schimenti J.C., et al. Mutations in Col4a1 cause perinatal cerebral hemorrhage and porencephaly. Science, 2005 May 20, 308(5725):1167-71.

11. Pöschl E., Schlötzer-Schrehardt U., Brachvogel B., Saito K., Ninomiya Y., Mayer U. Collagen IV is essential for basement membrane stability but dispensable for initiation of its assembly during early development. Dev Camb Engl, 2004 Apr, 131(7):1619-28.

12. Vermeulen R.J., Peeters-Scholte C., Van Vugt J.J.M., Van Vught J.J.M.G., Barkhof F., Rizzu P., et al. Fetal origin of brain damage in 2 infants with a COL4A1 mutation: fetal and neonatal MRI. Neuropediatrics, $2011 \mathrm{Feb}, 42(1): 1-3$.

13. Lichtenbelt K.D., Pistorius L.R., De Tollenaer S.M., Mancini G.M., De Vries L.S. Prenatal genetic confirmation of a COL4A1 mutation presenting with sonographic fetal intracranial hemorrhage. Ultrasound Obstet Gynecol, 2012 Jun 1, 39(6):726-7.

14. Garel C., Rosenblatt J., Moutard M.L., Heron D., Gelot A., Gonzales M., et al. Fetal intracerebral hemorrhage and COL4A1 mutation: promise and uncertainty. Ultrasound Obstet Gynecol, 2013 Feb 1, 41(2):228-30.

15. Strigini F.A.L, Cioni G., Canapicchi R., Nardini V., Capriello P., Carmignani A. Fetal intracranial hemorrhage: is minor maternal trauma a possible pathogenetic factor? Ultrasound Obstet Gynecol, 2001 Oct 1, 18(4):335-42. 